\title{
Review of structured guides for deprescribing
}

\author{
Ian Scott, ${ }^{1,2}$ Kristen Anderson, ${ }^{2}$ Christopher Freeman ${ }^{3}$
}

- Additional material is published online only. To view please visit the journal online (http://dx.doi.org/10.1136/ ejhpharm-2015-000864).

'Department of Internal Medicine and Clinical Epidemiology, Princess Alexandra Hospital, Woolloongabba, Queensland, Australia

${ }^{2}$ Centre of Research Excellence in Quality \& Safety in Integrated Primary-Secondary Care, School of Medicine, The University of Queensland, Brisbane, Australia

${ }^{3}$ Charming Institute, Brisbane, Queensland, Australia

Correspondence to Associate Professor lan A Scott, Department of Internal Medicine and Clinical Epidemiology, Princess Alexandra Hospital, Brisbane 4102, Australia; ian.scott@ health.qld.gov.au

Received 21 December 2015 Revised 28 February 2016 Accepted 22 March 2016

\section{ABSTRACT}

Avoiding inappropriate polypharmacy has become increasingly recognised as a safety imperative for older patient care. Deprescribing is an active process of reviewing all medications being used by individual patients that prompts clinicians to consider which medications have unfavourable risk-benefit trade-offs in the context of illness severity, advanced age, multimorbidity, physical and emotional capacity, life expectancy, care goals and personal preferences. Structured guides to deprescribing include algorithms, flow charts or tables which are patient-directed and aim to guide the clinician through sequential steps in deciding which medications should be targeted for discontinuation. In this narrative review, we describe seven structured deprescribing guides whose stated purpose included the reduction of polypharmacy, their use was not restricted to a single drug or drug class and they had undergone some form of efficacy testing. There was considerable heterogeneity in guide design and content, with some guides constituting little more than a set of principles while others entail detailed processes and sub-steps which addressed multiple determinants of drug appropriateness. Evidence of effectiveness for each guide was limited in that none have been evaluated in randomised controlled trials, that pilot or feasibility studies have involved relatively small patient samples, that intra-rater and inter-rater reliabilities have not been determined and that most have been studied in hospital settings. Which is most useful to clinicians is unknown in the absence of headto-head comparisons. While most guides have face validity, more research is needed for determining effectiveness and ease of use in routine clinical practice, especially in primary care settings.

\section{INTRODUCTION}

More than a third of older adults aged over 65 years use more than five prescription medicines and up to a quarter use eight or more. ${ }^{1}$ While many of these medications may confer benefit, they also increase risk of adverse drug effects, with the magnitude of risk directly related to the number of medications used. ${ }^{2}$ The avoidance of inappropriate polypharmacy has become recognised as a safety imperative for older patient care. ${ }^{3}$ Studies have estimated that at least one in five and up to one in three medicines used by older patients are potentially inappropriate. ${ }^{4}$ Almost two-thirds of hospitalised older patients are prescribed one or more potentially inappropriate medications (PIMs). ${ }^{6}$ These medications serve as targets for the process termed deprescribing. This is an active review process that prompts clinicians to consider which medications have lost their advantage in the harmbenefit trade-off, especially in patients with changing goals of care or limited life expectancy. ${ }^{7}$
Various instruments have been developed to assist the deprescribing process:

1. Screening tools or criteria that help identify medications more likely than not to be inappropriate for a given set of circumstances. These include the Beers criteria, ${ }^{8}$ STOPP tool, ${ }^{9}$ Improved Prescribing in the Elderly Tool, ${ }^{10}$ McLeod criteria, ${ }^{11}$ Medication Inappropriateness Index, ${ }^{12}$ Fit for the Aged Criteria $^{13}$ and the PRICUS list. ${ }^{14}$ The Beers criteria and STOPP tool are widely used and demonstrate utility in identifying PIMs in older patients. $^{15}$

2. Risk scales which estimate the global anticholinergic and sedative burden of all the medications being used by individual patients. These include the Drug Burden Index ${ }^{16}$ and the Anticholinergic Risk Scale, ${ }^{17}$ both of which strongly correlate anticholinergic drug effects with reduced physical and cognitive decline. ${ }^{18} 19$ However, their complexity has limited widespread use in clinical practice.

3. Risk scores ${ }^{20-23}$ or clinical prediction rules ${ }^{24}$ which estimate the risk of adverse drug events (ADEs) in individual patients based on multivariable regression models which include age, number of medications, renal function and comorbidities.

4. Deprescribing guidelines directed at particular medications (or drug classes) which identify clinical scenarios where a particular drug is likely to be inappropriate and how to safely wean or discontinue it. Such guidelines are proliferating, ${ }^{25-27}$ although their effects on clinical decision-making are yet to be evaluated.

All these instruments have limitations when used to assess the inappropriateness of all medications used by individual patients. They do not accommodate multiple factors which determine drug appropriateness such as illness severity, advanced age, multi-morbidity, physical and emotional capacity, life expectancy, care goals and personal preferences. ${ }^{7}$ Deciding which medications can be ceased in such a personalised context requires a highly nuanced approach. For example, while explicit, scenario-specific guides such as Beer's ${ }^{8}$ and STOPP criteria ${ }^{9}$ can identify 'medications to avoid' (such as potent opioids and non-steroidal anti-inflammatory medications); such medications account for less than $25 \%$ of all ADEs. ${ }^{28}$ Commonly prescribed 'non-Beers list' medications with proven benefits in many older people, such as cardiovascular medications, anticoagulants and hypoglycaemic agents, are more frequently implicated as a result of misuse. ${ }^{29}$

Up to half of the medications used by frail older patients who are entering residential aged care facilities from hospital comprise preventive medications which are probably inappropriate or even 
futile at the end of life. ${ }^{30} 31$ A patient who has received an antihypertensive or nitrates when still independent and active may not need these medications years later when disabled and exerting minimal physical effort. These examples argue for a more holistic, implicit and dynamic framework by which various determinants of medication appropriateness can be considered concurrently in tailoring medications to individual patient circumstances.

Structured guides to deprescribing include algorithms, flow charts or tables aimed at guiding the clinician through sequential steps in deciding which medications used by an individual patient should be targeted for discontinuation after due attention to all relevant factors. They take account of the complexity of polypharmacy and prompt a more systematic appraisal of all medications being used. This article reviews structured deprescribing guides as reported in published literature and comments on evidence supporting their use.

\section{METHODS}

A search was undertaken between 10 and 12 December 2015 of PubMed, Cochrane Library and CINAHL for relevant articles published between 1 January 1990 and 10 December 2015 (either in print or on-line) using search terms such as 'deprescribing' or 'discontinuation' or 'withdrawal' AND 'medicines', 'medications', AND 'algorithm' or 'flowchart' or 'checklist' or 'tables' or 'guides'. Reference lists of retrieved articles and the PubMed 'related articles' function were used to identify additional reports. Articles were screened for inclusion by one author (IS) and selected for analysis (confirmed by two other authors (KA and CF)) if the stated purpose of the guide included deprescribing, its use was not restricted to a single drug or drug class and it had been subjected to some form of efficacy testing. Data were collected (by IS) and confirmed (by KA) relating to year of publication, patient population, setting, guide derivation and validation and measures of efficacy. The marked study heterogeneity precluded pooling of data across studies and hence results are reported in narrative form.

\section{RESULTS}

Of the 186 retrieved articles, 15 reports relating to seven algorithms satisfied selection criteria and are discussed in more detail below.

\section{Geriatric medication evaluation algorithm}

This algorithm (figure 1) was designed by five academic internists in a hospital general medicine outpatient setting. It targeted high-risk medications considered by expert geriatricians to be strongly associated with exacerbations of common diseases in the elderly or causing excessive side effects. ${ }^{32}$ The algorithm was tested in an outpatient clinic of a US tertiary hospital where the medications of a convenience sample of 41 patients aged over 65 years taking more than three medications were compared pre-algorithm and post-algorithm. The average (SD) number of medications pre-algorithm was 5.8 (1.62) per patient which reduced to $5.6(1.69)$ post-algorithm, a difference of 0.3 (0.67), $\mathrm{p}<0.025$. Of the 22 medications discontinued, 9 medications (44\%) were high risk compared with a baseline prevalence of $21 \%$.

\section{The good palliative-geriatric algorithm}

This geriatrician-led team developed this algorithm as a consensus-based flow chart for reducing polypharmacy developed for patients in Israeli nursing care facilities (figure 2). ${ }^{33}$ It asks if an evidence base exists suggesting benefit of the drug in its current dose in a patient's age group, given disability levels, and whether such benefits outweigh all known adverse effects. If the answer is no to both, then the drug should be discontinued. Additionally, if adverse effects are noted, which could be related to a drug otherwise considered to be beneficial, it should be changed to a superior drug or continued but at a reduced dose.

The algorithm was first evaluated in an intervention group of 119 disabled patients in six geriatric nursing departments and compared with 71 control patients of comparable age, gender and comorbidities within the same wards under the same treating physicians. ${ }^{33}$ After 12 months follow-up, 332 different medications were discontinued among intervention patients (mean 2.8 medications per patient) without any significant adverse effects, compared with no change among controls. The overall rate of drug discontinuation failure was $18 \%$ of all patients and $10 \%$ of all medications. The 12-month unadjusted mortality rate was $45 \%$ in the control group compared with $21 \%$ in the intervention group $(\mathrm{p}<0.001)$, while the annual referral rate to acute care facilities was $30 \%$ versus $12 \%(\mathrm{p}<0.002)$.

A second prospective cohort study involved 70 consecutive community-dwelling older patients referred to a hospital geriatric clinic by family physicians or family for comprehensive geriatric assessment. ${ }^{34}$ Patients were of mean (SD) age 82.8 years (6.9) and using a mean (SD) of 7.7 (3.7) medications. The algorithm recommended discontinuation of 4.4 (2.5) medications per patient, comprising 311 medications in 64 patients $(58 \%$ of all medications). Successful discontinuation was achieved in $81 \%$ of instances. No significant adverse events or deaths were attributable to discontinuation and $88 \%$ of patients reported global improvement in health.

\section{Prescribing optimisation method}

This method (box 1) incorporates six questions relating to undertreatment, compliance, medications that can be discontinued, ADEs, interactions and dosing frequency or administration. ${ }^{35}$ In an evaluation study, 45 general practitioners (GPs) were asked to optimise the medications of two case histories, randomly chosen from 10 histories of patients seen at a hospital geriatric outpatient clinic. Patients suffered a mean (SD) of 7.9 (1.2) problems treated with 8.7 (3.1) medications. The first case was optimised without knowledge of the prescribing optimisation method (POM); following a $2 \mathrm{~h}$ lecture on the POM, the GPs used the POM to optimise the medication of the second case history over $20 \mathrm{~min}$. Medication decisions were compared with those made by an expert panel of four geriatricians specialised in clinical pharmacology. The proportion of correct decisions increased from $34.7 \%$ without the POM to $48.1 \%$ with the POM $(p=0.004)$ and the number of potentially harmful decisions decreased from 3.3 (1.8) per case without the POM to 2.4 (1.4) with the POM $(\mathrm{p}=0.005)$.

\section{Assess, review, minimise, optimise, reassess}

This method (table 1) is a functional, interactive tool designed for use in US long-term care facilities in Michigan, USA. ${ }^{36}$ It considers the patient's clinical profile and functional status and existing evidence of benefit versus harm. Restoration and maintenance of functional status are the primary outcome goals. Quality of life is considered a key factor in deciding to discontinue medications which may adversely impact on primary biological functions such as bladder, bowel and appetite. It was designed to be used in patients: (1) receiving nine or more medications; (2) with falls or behavioural disturbance and/or (3) admitted for rehabilitation. The authors report their experience 
I. 1. Obtain complete medication list from patient

2. Obtain orthostatic blood pressure

II. Evaluate each drug

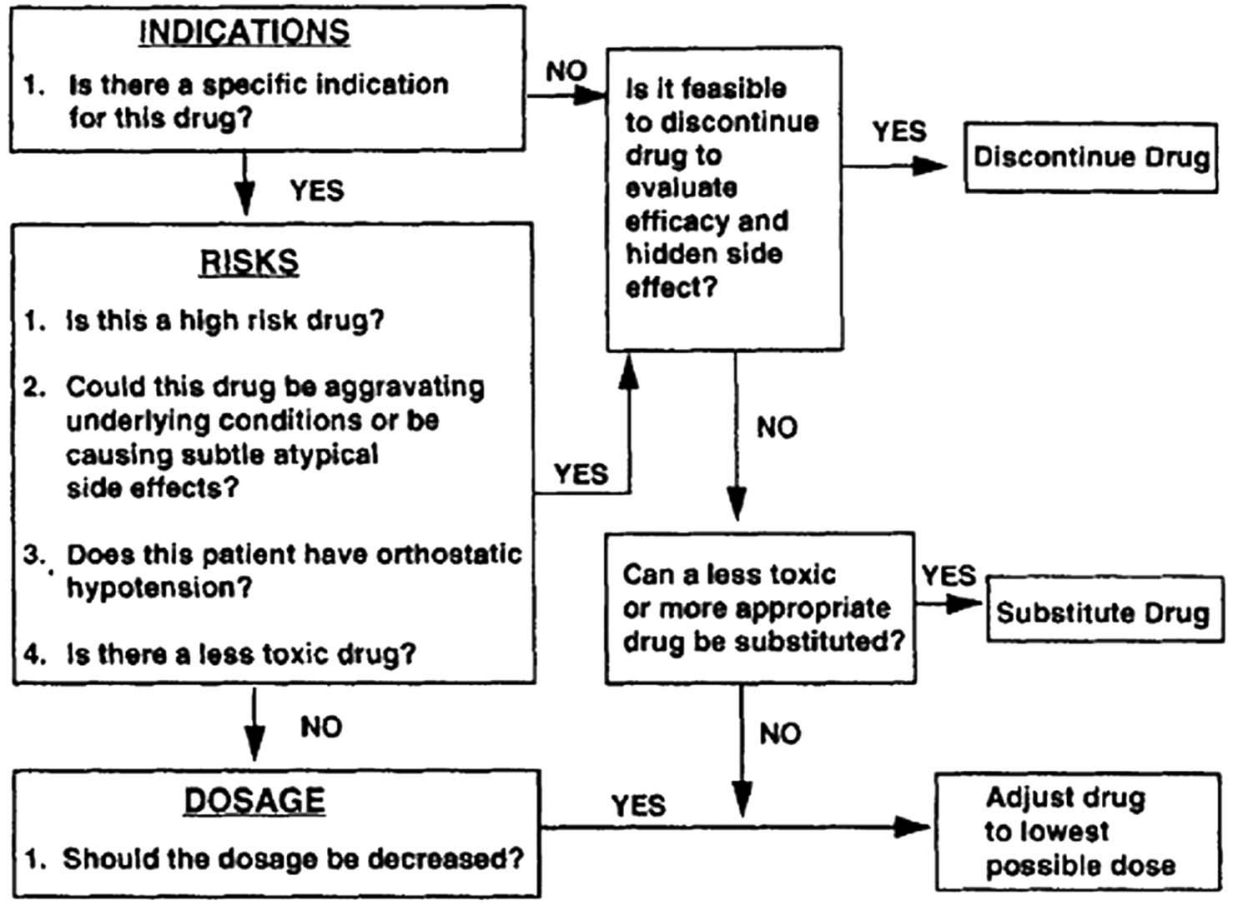

\section{Evaluate Entire Drug Regimen}

\section{Are there drug interactions or} additive side effects?

2. Could the drug regimen be simplifled?

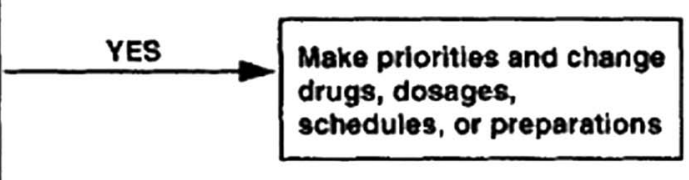

IV. Evaluate Compliance

1. Is the patient/caregiver unclear about the medication regimen?

2. Does the patient need a compliance aid?

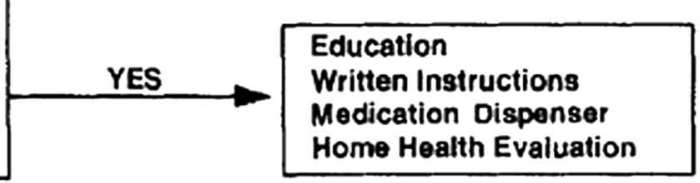

Figure 1 The Geriatric Medication Evaluation Algorithm ${ }^{32}$ (Reproduced with permission of the Society of General Internal Medicine).

in using the method based on routinely collected data although no numerical results are reported. They noted a clear and consistent decline in the use of nine or more medications over a 6-month period, including antipsychotics and antidepressants, compared with state and national averages (noted from Minimum Data Set (MDS) coordination feedback from state regulatory oversight). A reduction in falls and behaviours (with potential harm to self and others) was also seen in their inpatient facility.

\section{Geriatric Risk Assessment MedGuide}

This is a clinical informatics tool that generates prospective monitoring plans based on potential risk of falls or for delirium within $24 \mathrm{~h}$ of admission to a nursing home. ${ }^{37}$ It was developed by the American Society of Consultant Pharmacists Foundation and its face and content validity were confirmed by an interdisciplinary panel of experts in geriatric care. The Geriatric Risk Assessment MedGuide (GRAM) software is an enhanced version of the MDS -MedGuide ${ }^{38}$ and correlates medication effects with physical, functional and cognitive decline to foster early recognition of potential adverse drug effects and an escalation in care which includes medication discontinuation. Two types of GRAM reports are generated based on the residents' medications: GRAM RAP-Med report, which identified resident-specific medications that potentially cause, aggravate or contribute to delirium and fall risk (separately) and Medication Monitoring Care Plans and Flow Records, one for delirium and the other for falls.

The GRAM was evaluated in a cluster-randomised trial involving 25 US nursing homes and 6523 residents in Ohio, USA, serviced by two long-term care pharmacies. ${ }^{37}$ GRAM triggered monitoring plans for 491 residents. The total numbers of medications received per patient did not differ significantly between intervention and usual care nursing home groups before and after GRAM implementation. The former saw a 3\% and $4 \%$ absolute reduction in use of opiate/anticonvulsant and tranquiliser medications, respectively. Hospitalisations due to potential ADEs declined $32 \%$ in intervention homes versus $14 \%$ in usual care homes. 
Figure 2 The Good PalliativeGeriatric Practice algorithm ${ }^{33}$

(Reproduced with permission of the Israeli Medical Association).
Discuss the following with the patient/guardian

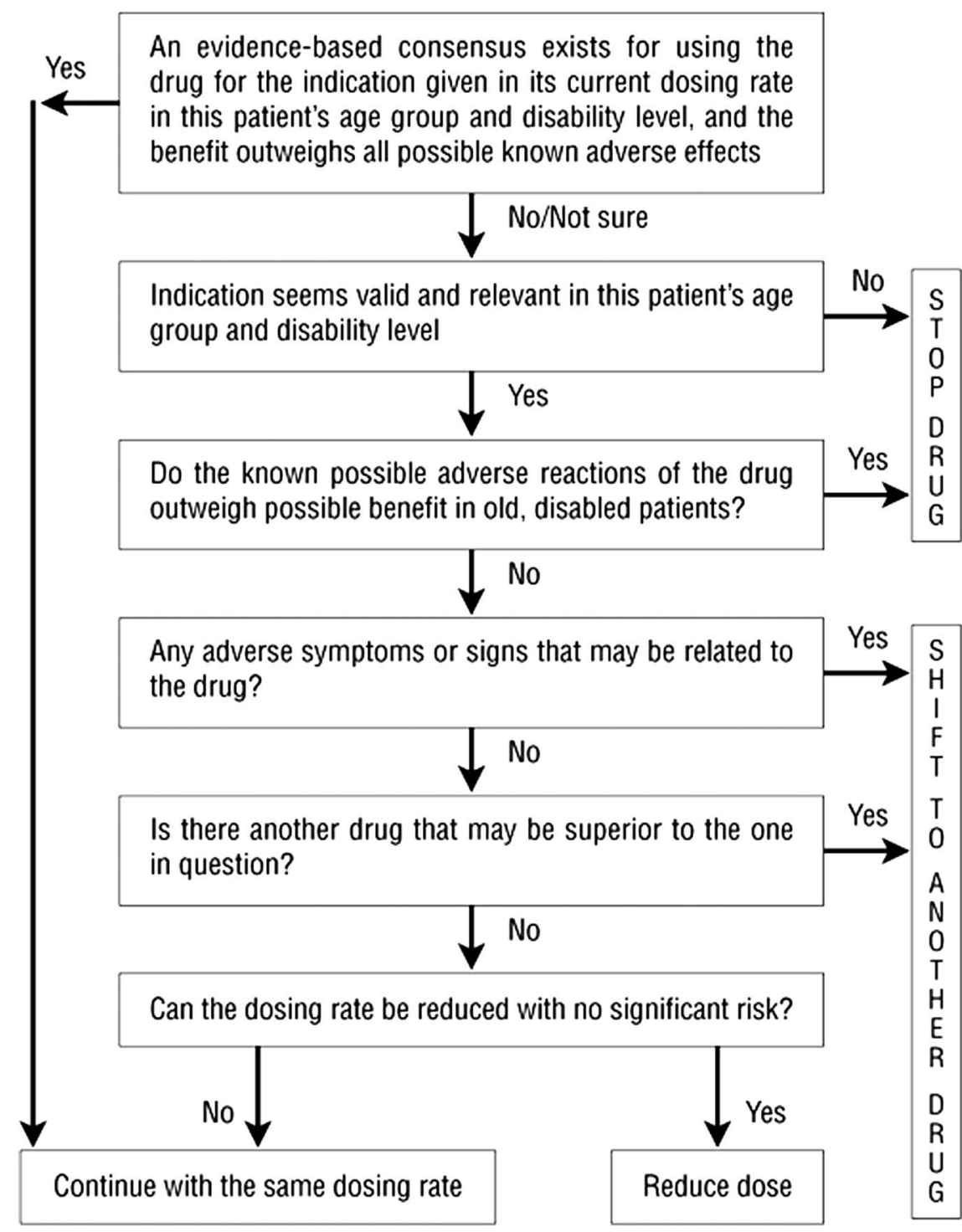

\section{Medication algorithms for reducing polypharmacy in mental health}

Two studies focused on medication algorithms for patients with mental health disorders, both developed by local experts based on published evidence with reference to the Texas Medication Algorithm Project. ${ }^{39}$ The first study involved adult patients admitted to a psychiatric unit of a regional hospital in the

\section{Box 1 Prescribing optimisation method ${ }^{35}$}

1. Is the patient undertreated and is additional medication indicated?

2. Does the patient adhere to his/her medication schedule?

3. Which medications(s) can be withdrawn or which drug(s) is/ are inappropriate for this patient?

4. Which adverse effects are present?

5. Which clinically relevant interactions are to be expected?

6. Should the dose frequency and/or form of the drug be adjusted?
USA. ${ }^{40}$ The algorithm attempted to optimise single-drug regimens, where appropriate, and to eliminate redundant or ineffective medications. Information from patients, families and practitioners was used to review adequacy of previous trials and the degree of improvement, tolerability and adherence associated with each current medication. A feasibility pilot study was conducted using a matched case-control design involving 12 patients managed with the algorithm and 12 historical controls matched according to diagnosis, number of medications on admission and age. A significant decrease was seen in the mean (SD) number of psychotropic medications per case at hospital discharge from $3.7(1.0)$ to $2.2(0.9, \mathrm{p}=0.003)$ compared with no change in the controls (3.6 (1.0) vs $3.7(1.0))$.

The second study involved adult patients admitted to an early psychosis intervention programme within a Singaporean tertiary hospital. ${ }^{41}$ It evaluated a treatment algorithm (not reproduced in the report) using a case-control design wherein prescriptions following use of the algorithm among 483 cases were compared with 68 historical controls matched for age and gender. The algorithm was associated with a greater proportion of intervention patients receiving only one antipsychotic drug (from $77 \%$ to $92 \%$ ) and a decrease in the proportions taking two or more 


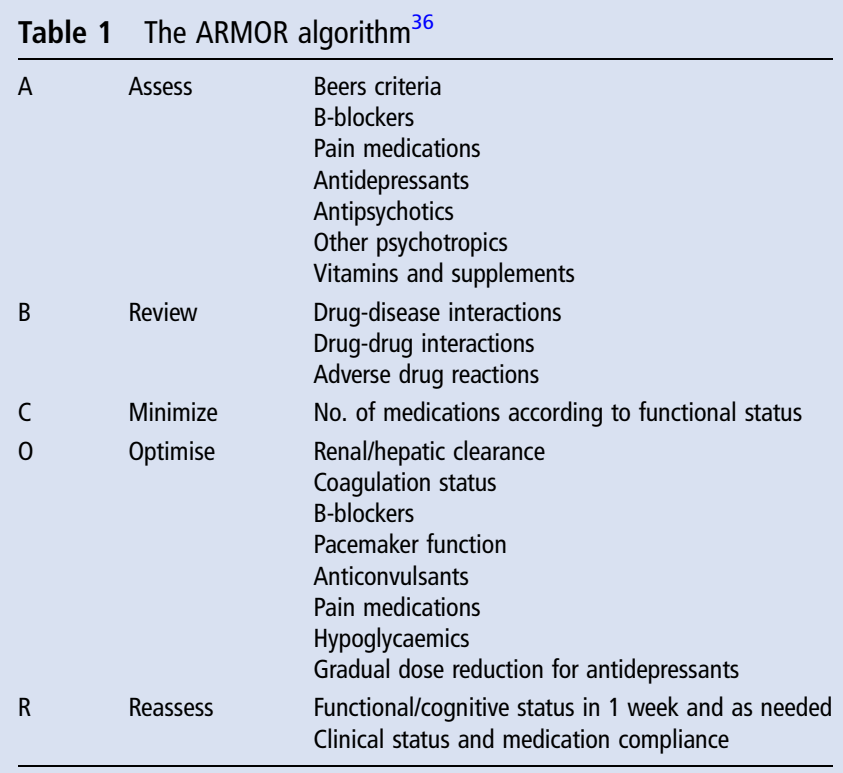

( $23 \%$ to $5 \%, \mathrm{p}<0.05$ for both comparisons) versus no significant change in controls.

\section{Confirm, estimate, assess, sort, eliminate}

This five-step protocol (box 2$)^{42}$ is the summary form of several other guides developed by the same group of researchers with expertise in gerontological prescribing. The original 10-step conceptual framework ${ }^{43}$ was used to derive a 10-step algorithm (figure 3$)^{44}$ which was further refined to a five-step guide with detailed sub-steps around issues that required consideration. ${ }^{42}$

The protocol has been evaluated in two uncontrolled studies involving hospital staff and patients. The first involved 61 clinicians who were presented with clinical information about a hypothetical case: an 81-year-old female with 12 chronic diseases, receiving 19 different medications. ${ }^{45}$ On a standardised, anonymous form, each participant indicated, as a pretest, which medications they felt strongly inclined to discontinue or to continue. The five-step guide was then presented and applied to the case, and participants repeated the drug selection process. Among the entire cohort, the mean (SD) number of medications selected for discontinuation increased from 6.0 (2.7) pretest to $9.6(3.2)$ post-test $(\mathrm{p}<0.001)$, with the greatest increases seen among consultant physicians $(6.6(2.3)$ to 11.5 $(2.9), \mathrm{p}<0.001)$ and clinical pharmacists $(5.3(2.6)$ to $8.9(2.2)$, $\mathrm{p}<0.001)$.

In a prospective study involving 50 hospitalised patients of median age 83 years with six comorbidities and receiving eight or more medications, application of the algorithm led to discontinuation of 186 of 542 regular medications $(34.3 \%) .{ }^{46}$ This represented a significant decrease in the median (IQR) number of medications per patient at discharge (7 (5-9)) compared to that at presentation $(10(9-12 \mathrm{p}<0.001))$. Statins, gastric acid suppressive agents, ACE inhibitors/angiotensin receptor antagonists and inhaled bronchodilators were the most frequently ceased medications. 39 of the 50 patients had follow-up status ascertained at a median of 78 days, only in whom 5 of 413 $(1.2 \%)$ ceased medications were recommenced among three patients due to symptom relapse. There were no instances of readmissions or ADEs as a result of deprescribing. The protocol is currently being evaluated in a controlled pilot study involving 20 GPs.
Box 2 Confirm, estimate, assess, sort, eliminate (CEASE) protocol $^{42}$

- Confirm current medicines-ascertain all medicines the patient is currently taking and the reasons for each one (also termed medication reconciliation).

- Estimate risk-consider the potential for this patient to be harmed by the medicines being prescribed in determining required intensity of deprescribing intervention:

- Consider risk factors such as total number of medications, age, presence of medications associated with high risk (eg, opiates, benzodiazepines, psychotropics, anticoagulants, hypoglycaemic medications, cardiovascular medications), past non-adherence, multiple prescribers, impaired cognition and poor social support, substance abuse, mental health problems.

- Assess each medicine for its usefulness in relation to its risk by considering:

- Indications for the drug (is the continued prescribing of the drug justified on the basis of a verified diagnosis and robust evidence of effectiveness for this indication in this patient?);

- Effects of the drug to date on the underlying disease process and/or its symptoms;

- Future benefit-harm trade-offs in the context of life expectancy, time until benefit (for preventive medications), goals of care (symptom relief vs disease modification vs cure) and patient values and preferences.

- Sort-prioritise those medicines for discontinuation with lowest utility (or highest disutility) and greatest ease of discontinuation, while taking patient preferences into account.

- Eliminate-implement a discontinuation regimen and monitor patients closely for improvement in outcomes or onset of withdrawal or rebound syndromes.

A modified form of the algorithm (see online supplementary figure) targets patients in residential aged care facilities and comprises sequential steps: (1) identifying a high-risk medication; (2) ascertaining its current indications and assessing their validity; (3) assessing if the drug is providing ongoing symptomatic benefit and (4) considering withdrawing, altering or continuing it. ${ }^{47}$ Decision support resources complementing the algorithm include lists of high-risk medications and the reasons for inappropriateness, alternative treatments and suggested medication withdrawal protocols. The utility and clinical impact of the algorithm is currently being assessed in a cluster-randomised trial of geriatric telehealth consultations involving eight residential aged care facilities.

\section{DISCUSSION}

This review of structured deprescribing guides demonstrates that the guides are relatively few in number and have limited evidence of effectiveness. Only two have been tested in intervention trials using concurrent controls; ${ }^{33} 37$ pilot or feasibility studies have involved relatively small patient samples, intra-rater and inter-rater reliabilities have not been determined and most have been studied as specialist-mediated tools in hospital and residential aged care settings. As a result, estimates of effectiveness may be inflated by surveillance bias compared with their use in primary care. Only one guide (or its derivatives) has been 


\section{Accurately ascertain all current drug use \\ - 'brown paper bag' medication reconciliation}

2. Identify patients at risk of, or suffering, $A D R$

- at risk: $\geq 8$ medications advanced age ( $>75$ years) high-risk medications

- assess for current, past or highly likely future toxicity

3. Estimate life expectancy

- clinical prognostication tools or lifespan calculators

4. Define overall care goals

- consider current functional status and quality of life with Ireference to estimated life expectancy

5. Verify current indications for ongoing treatments

- perform diagnosis-medication reconciliation

- confirm diagnostic labels against formal diagnostic criteria

- ascertain, for each confirmed diagnosis, drug appropriateness $\downarrow$

6. Determine need for disease-specific preventive medications

- estimate clinical impact and time to future treatment benefit

- compare this estimate with expected lifespan

7. Determine absolute benefit-harm thresholds of medications

- reconcile estimates of absolute benefit and harm using prediction ltools (see http://www.mdcalc.com)

8. Review the relative utility of individual drugs

- rank drugs according to the relative utility from high to low based on predicted benefit, harm, administration and monitoring burden

9. Identify drugs to be discontinued and seek patient consent

- reconcile drugs for discontinuation with patient preferences<smiles>[123IH]</smiles>

10. Devise and implement drug discontinuation plan with close monitoring

\section{All three at-risk criteria - aim for $\leq 5$ drugs \\ Discontinue drugs for which there is unequivoval \\ evidence of past, current or future toxicity \\ (eg triple whammy of NSAID, diuretic, ACE inhibitor)}

\section{If life expectancy less than 2 years, preservation of function and quality of life predominate over \\ .... prolonging life and avoiding future complications as goals of care \\ Discontinue drugs for which the diagnosis is wrong or totally unsubstantiated or where, for a confirmed diagnosis, the drug is ineffective}
.... Discontinue preventive drugs whose time until benefit exceeds expected lifespan

\section{.... Discontinue drugs whose absolute level of harm exceeds absolute level of benefit; in 'line-ball' cases elicit patient preferences}

Discontinue drugs of low utility

Discontinue drugs patients are not in favour of taking

Figure 3 10-step deprescribing algorithm ${ }^{44}$ (Reproduced with permission British Medical Journal Group).

designed for use in multiple settings. ${ }^{42}$ There is considerable heterogeneity in guide design and content, with some guides constituting little more than a set of principles, ${ }^{35} 36$ while others entail detailed processes and sub-steps. ${ }^{3742}$ Some guides cover the spectrum of optimal prescribing, including initiation and titration of indicated medications, ${ }^{35}$ while others are dedicated solely to discontinuation of PIMs. ${ }^{40-42}$ Determining which guides are most useful to clinicians requires head-to-head comparisons which enrol participants with varying levels of confidence and competence in deprescribing. ${ }^{48}$

The strongest evidence to date of efficacy and clinician acceptability is seen for the Good Palliative-Geriatric Algorithm ${ }^{33}$ (with the advantage of a controlled trial evaluation) and the confirm, estimate, assess, sort, eliminate (CEASE) guide. ${ }^{42}$ Both have been subject to a process of development and refinement over months to years involving multiple clinician prescribers and pharmacists. However, the former was designed in nursing home settings ${ }^{33}$ and then applied to a community-based population $^{34}$ without further validation, whereas the CEASE protocol has assumed different forms according to the needs of different settings. ${ }^{46} 47$

In terms of effects in reducing polypharmacy, some guides listed here appear to achieve reductions in the number of inappropriate medications prescribed (between three ${ }^{42}$ and four $^{34}$ per patient) which are comparable with, or exceed, those of other strategies such as prescriber feedback, ${ }^{14}{ }^{49}$ geriatric assessment, ${ }^{14}$ pharmacist medication reviews, ${ }^{50}$ clinician education and training $^{14}$ or multifaceted interventions. ${ }^{51}{ }^{52}$
Structured guides offer a logical sequence of clear-cut decision steps which are easy to assimilate and apply to patient care. In time they may be incorporated into computerised decision support systems and linked automatically with pathology and pharmacy databases in ways that deliver a high level of sophistication in minimising use of PIMs in individual patients. ${ }^{53}$

Our review has several limitations. Our literature search may not have retrieved all relevant articles as deprescribing, as an index search term, has only recently been formalised. ${ }^{54}$ However, several synonymous terms and linkage functions for related articles were used across three large electronic databases. Information on exactly how algorithms were derived and validated, how reliable and time-efficient they are in routine care and when and how different clinicians (specialists, GPs, pharmacists) choose to use them was missing from most reports. More qualitative research is needed in optimising the design, format and method of presentation (paper, electronic) of the guides for use by different clinician groups.

In conclusion, while our review of deprescribing guides may not be exhaustive, it does provide an indication of the current state of the art and the growing interest of researchers to develop and evaluate these tools in addressing the growing burden of inappropriate polypharmacy in clinical practice.

Contributors IAS conceived the concept, undertook literature search and wrote the first draft of the manuscript; KA and CF assisted in confirming article selection, KA cross-checked extracted data from relevant articles and KA and CF critically reviewed the manuscript. 
Competing interests None declared.

Provenance and peer review Not commissioned; externally peer reviewed.

\section{REFERENCES}

1 Hajjar ER, Cafiero AC, Hanlon JT. Polypharmacy in elderly patients. Am J Geriatr Pharmacother 2007;5:345-51.

2 Steinman MA, Miao Y, Boscardin WJ, et al. Prescribing quality in older veterans: a multifocal approach. J Gen Intern Med 2014;29:1379-86.

3 Kalisch LM, Caughey GE, Barratt JD, et al. Prevalence of preventable medication-related hospitalizations in Australia: an opportunity to reduce harm. Int J Qual Health Care 2012;24:239-49.

4 Roughead EE, Anderson B, Gilbert AL. Potentially inappropriate prescribing among Australian veterans and war widows/widowers. Intern Med J 2007;37:402-5.

5 Stafford AC, Alswayan MS, Tenni PC. Inappropriate prescribing in older residents of Australian care homes. J Clin Pharm Ther 2011;36:33-44.

6 Wahab MS, Nyfort-Hansen K, Kowalski SR, Inappropriate prescribing in hospitalised Australian elderly as determined by the STOPP criteria. Int I Clin Pharm 2012;34:855-62.

7 Scott IA, Hilmer SN, Reeve $E$, et al. Reducing inappropriate polypharmacy - the process of deprescribing. JAMA Intern Med 2015;175:827-34.

8 American Geriatrics Society Beers Criteria Update Expert Panel. American Geriatrics Society updated Beers Criteria for potentially inappropriate medication use in older adults. J Am Geriatr Soc 2012;60:616-31.

9 Gallagher P, Ryan C, Byrne S, et al. STOP (Screening Tool of Older Person's Prescriptions) and START (Screening Tool to Alert doctors to Right Treatment). Consensus validation. Int J Clin Pharmacol Ther 2008;46:72-83.

10 Naugler CT, Brymer C, Stolee P, et al. Development and validation of an improving prescribing in the elderly tool. Can J Clin Pharmacol 2000;7:103-7.

11 McLeod PJ, Huang AR, Tamblyn RM, et al. Defining inappropriate practices in prescribing for elderly people: a national consensus panel. Can Med Assoc J 1997:156:385-91.

12 Hanlon JT, Schmader KE, Samsa GP, et al. A method for assessing drug therapy appropriateness. J Clin Epidemiol 1992;45:1045-51.

13 Wheling M. Multimorbidity and polypharmacy: how to reduce the harmful drug load and yet add needed medications in the elderly? Proposal of a new drug classification: fit for the aged. J Am Geriatr Soc 2009;57:560-1.

14 Holt S, Schmiedl S, Thürmann PA. Potentially inappropriate medications in the elderly: the PRISCUS list. Dtsch Arztebl Int 2010;107:543-51.

15 Patterson SM, Cadogan CA, Kerse N, et al. Interventions to improve the appropriate use of polypharmacy for older people. Cochrane Database Syst Rev 2014;10: CD008165.

16 Hilmer SN, Mager DE, Simonsick EM, et al. A drug burden index to define the functional burden of medications in older people. Arch Intern Med 2007;167:781-7.

17 Rudolph JL, Salow MJ, Angelini MC, et al. The anticholinergic risk scale and anticholinergic adverse effects in older persons. Arch Intern Med 2008;168:508-13.

18 Lowry E, Woodman RJ, Soiza RL, et al. Associations between the anticholinergic risk scale score and physical function: potential implications for adverse outcomes in older hospitalised patients. J Am Med Dir Assoc 2011;12:565-72.

19 Lowry E, Woodman RJ, Soiza RL, et al. Drug burden index, physical function, and adverse outcomes in older hospitalised patients. J Clin Pharmacol 2012;52:1584-91.

20 Onder G, Petrovic M, Tangiisuran B, et al. Development and validation of a score to assess risk of adverse drug reactions among in-hospital patients 65 years or older: the GerontoNet ADR risk score. Arch Intern Med 2010;170:1142-8.

21 Urbina 0, Ferrandez O, Grau S, et al. Design of a score to identify hospitalized patients at risk of drug-related problems. Pharmacoepidemiol Drug Saf 2014;23:923-32

22 Trivalle C, Burlaud A, Ducimetiere P. Risk factors for adverse drug events in hospitalized elderly patients: a geriatric score. Eur Geriatr Med 2011:2:284-9.

23 Saedder EA, Lisby M, Nielsen LP, et al. Detection of patients at high risk of medication errors: development and validation of an algorithm. Basic Clin Pharmacol Toxicol 2016;118:143-9.

24 Sakuma M, Bates DW, Morimoto T. Clinical prediction rule to identify high-risk inpatients for adverse drug events: the JADE study. Pharmacoepidemiol Drug Saf 2012;21:1221-6.

25 No authors listed. A practical guide to stopping medicines in older people. http:/ www.bpac.org.nz/magazine/2010/april/stopGuide.asp

26 Lindsay J, Dooley M, Martin J, et al. The development and evaluation of an oncological palliative care deprescribing guideline: the 'OncPal deprescribing guideline'. Support Care Cancer 2015:23:71-8.

27 Conklin J, Farrell B, Ward N, et al. Developmental evaluation as a strategy to enhance the uptake and use of deprescribing guidelines: protocol for a multiple case study. Implement Sci 2015;10:91-101.
28 Miller GC, Valenti L, Britt $\mathrm{H}$, et al. Medications causing adverse events in patients aged 45 or older: a randomised survey of Australian general practice patients. BMJ Open 2013;3:e003701.

29 Budnitz DS, Lovegrove MC, Shehab N, et al. Emergency hospitalizations for adverse drug events in older Americans. N Engl J Med 2011;365:2002-12.

30 Hopcroft P, Peel NM, Poudel A, et al. Prescribing for older people discharged from the acute sector to residential aged-care facilities. Intern Med J 2014;44: 1034-7.

31 Todd A, Holmes HM. Recommendations to support deprescribing medications late in life. Int J Clin Pharm 2015:37:678-81.

32 Newton PF, Levinson W, Maslen D. The geriatric medication algorithm: a pilot study. J Gen Intern Med 1994:9:164-7.

33 Garfinkel D, Zur-Gil S, Ben-Israel J. The war against polypharmacy: a new cost-effective geriatric-palliative approach for improving drug therapy in disabled elldery people. Is Med Assoc J 2007;9:430-4.

34 Garfinkel D, Mangin D. Feasibility study of a systematic approach for discontinuation of multiple medications in older adults: addressing polypharmacy. Arch Intern Med 2010;170:1648-54.

35 Drenth-van Maanen AC, van Marum RJ, Knol W, et al. Prescribing optimization method for improving prescribing in elderly patients receving polypharmacy: results of application to case histories by general practitioners. Medications Aging 2009;26:687-701.

36 Haque R. ARMOR: a tool to evaluate polypharmacy in elderly persons. Ann Long Term Care 2009;17:26-30. http://www.annalsoflongtermcare.com/content/ armor-a-tool-evaluate-polypharmacy-elderly-persons

37 Lapane KL, Hughes CM, Daiello LA, et al. Effect of a pharmacist-led multicomponent intervention focusing on the medication monitoring phase to prevent potential adverse drug events in nursing homes. J Am Geriatr Soc 2011;59:1238-45.

38 Tobias DE, Feinberg JL, Troutman WG. The MDS-Med Guide. Consult Pharm 1999;14:831-60

39 Rush AJ, Rago WV, Crismon ML, et al. Medication treatment for the severely and persistently mentally ill: The Texas medication algorithm project. J Clin Psychiatry 1999;60:284-91.

40 Mistler LA, Mellman TA, Drake RE. A pilot study testing a medication algorithm to reduce polypharmacy. Qual Saf Health Care 2009;18:55-8.

41 Chong SA, Ravichandran N, Poon LY, et al. Reducing polypharmacy through the introduction of a treatment algorithm: use of a treatment algorithm on the impact on polypharmacy. Ann Acad Med Singap 2006:35:457-60.

42 Scott IA, Le Couteur D. Physicians need to take the lead in deprescribing. Intern Med J 2015;45:352-6.

43 Scott IA, Martin JH, Gray LA, et al. Minimising inappropriate medications in older populations-a 10 step conceptual framework. Am J Med 2012;125:529-37.

44 Scott IA, Gray LA, Martin JH, et al. Deciding when to stop: towards evidence-based deprescribing of medications in older populations. Evidence-based Med 2013;18:121-4

45 Scott IA, Martin JH, Gray LA, et al. Effects of a drug minimisation guide on prescribing intentions in elderly persons with polypharmacy. Medications Ageing 2012;29:659-67.

46 McKean M, Pillans P, Scott IA. A medication review and deprescribing method for hospitalised older patients receiving multiple medications. Intern Med J 2016:46:35-42.

47 Poudel A, Ballokova A, Hubbard RE, et al. Algorithm of medication review in frail older people: Focus on minimizing the use of high-risk medications Geriatr Gerontol Int 2015. Sep 3. doi:10.1111/ggi.12589. [Epub ahead of print]

48 Kroenke K, Pinholt EM. Reducing polypharmacy in the elderly: a controlled trial of physician feedback. J Am Geriatr Soc 1990;38:31-6.

49 Castelino RL, Bajorek BV, Chen TF. Targeting suboptimal prescribing in the elderly: a review of the impact of pharmacy services. Ann Pharmacother 2009;43:1096-106

50 Thompson A, Sullivan SA, Barley M, et al. The DEBIT trial: an intervention to reduce antipsychotic polypharmacy prescribing in adult psychiatry wards - a cluster randomized controlled trial. Psychol Med 2008;38:705-15.

51 Spinewine A, Schmader KE, Barber N, et al. Appropriate prescribing in elderly people: how well can it be measured and optimised? Lancet 2007;370:173-84.

52 Anderson $\mathrm{K}$, Stowasser D, Freeman C, et al. Prescriber barriers and enablers to minimising potentially inappropriate medications in adults: A systematic review and thematic synthesis. BMJ Open 2014;4:e006544.

53 Sönnichsen A, Trampisch US, Rieckert A, et al. Polypharmacy in chronic diseases-Reduction of Inappropriate Medication and Adverse drug events in older populations by electronic Decision Support (PRIMA-eDS): study protocol for a randomized controlled trial. Trials 2016;17:57.

54 Alldred DP. Deprescribing: a brave new word? Int J Pharm Pract 2014;22:2-3. 